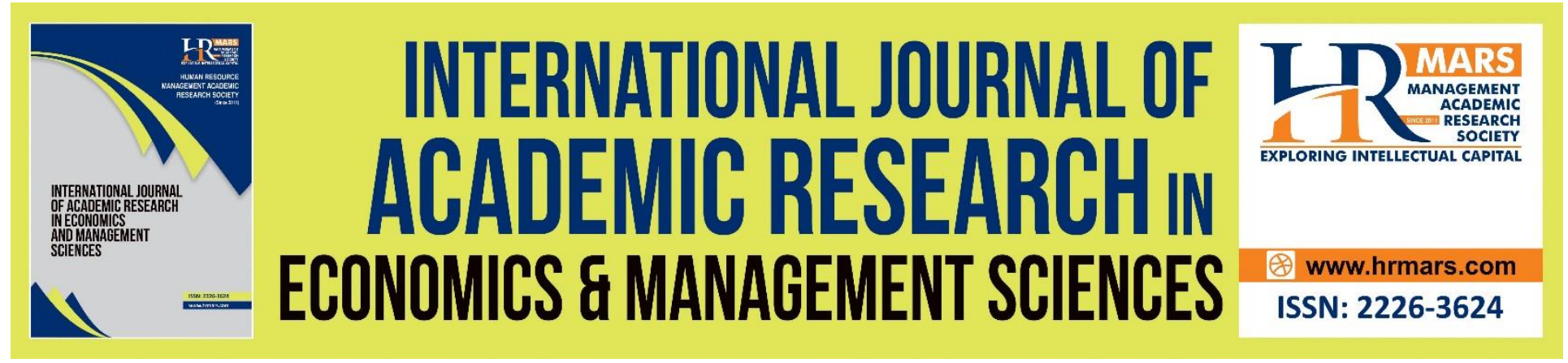

\title{
Timeliness of Quarterly Reports and Pandemic Disease
}

\section{Zarina Abdul Salam, Adriana Mohd Rizal, Maizaitulaidawati Md Husin}

To Link this Article: http://dx.doi.org/10.6007/IJAREMS/v10-i3/11366

DOI:10.6007/IJAREMS/v10-i3/11366

Received: 20 July 2021, Revised: 22 August 2021, Accepted: 06 September 2021

Published Online: 25 September 2021

In-Text Citation: (Salam et al., 2021)

To Cite this Article: Salam, Z. A., Rizal, A. M., \& Husin, M. M. (2021). Timeliness of Quarterly Reports and Pandemic Disease. International Journal of Academic Research in Economics and Management and Sciences, 10(3), 428-440.

\section{Copyright: (C) 2021 The Author(s)}

Published by Human Resource Management Academic Research Society (www.hrmars.com)

This article is published under the Creative Commons Attribution (CC BY 4.0) license. Anyone may reproduce, distribute, translate and create derivative works of this article (for both commercial and non-commercial purposes), subject to full attribution to the original publication and authors. The full terms of this license may be seen at: http://creativecommons.org/licences/by/4.0/legalcode

Vol. 10, No. 3, 2021, Pg. 428 - 440

http://hrmars.com/index.php/pages/detail/IJAREMS

JOURNAL HOMEPAGE

Full Terms \& Conditions of access and use can be found at http://hrmars.com/index.php/pages/detail/publication-ethics 


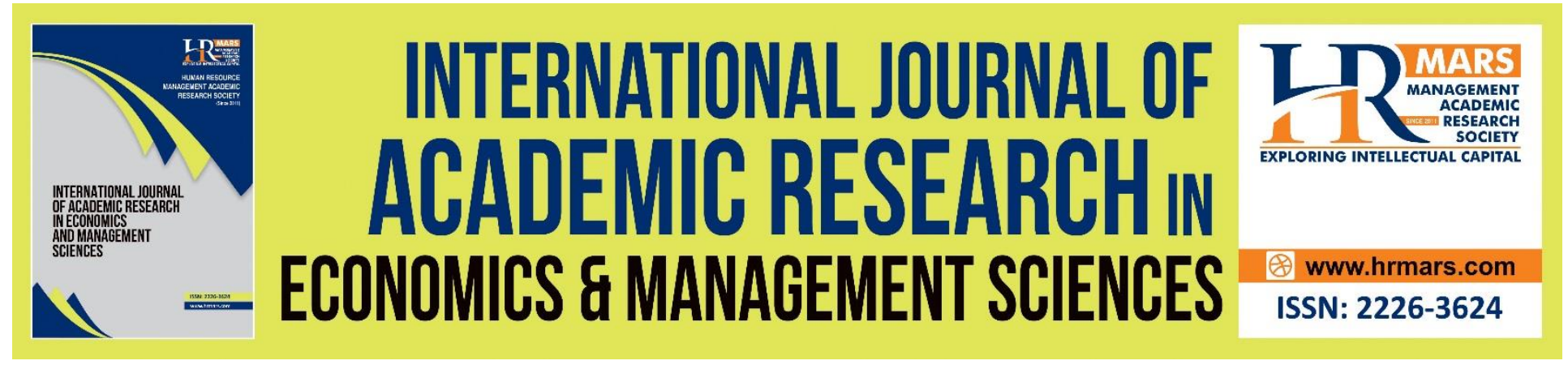

\title{
Timeliness of Quarterly Reports and Pandemic Disease
}

\author{
Zarina Abdul Salam, Adriana Mohd Rizal, Maizaitulaidawati Md \\ Husin \\ Azman Hashim International Business School, University Technology Malaysia
}

\begin{abstract}
This paper accentuates the importance of timeliness to issue quarterly reports using a sample of Malaysian public listed companies from all industries. Data were taken before and during the pandemic disease to investigate if there is any difference of timeliness to issue the quarterly reports. The data were analyzed by using statistical package for social science. Descriptive statistics and paired sample t-tests were used to answer the objectives of this study. The results suggested that some companies had problem in submitting the quarterly reports and took the opportunity to delay submission when the Bursa Malaysia granted one month extension in April 2020, just when the pandemic disease started. Implications and future research are discussed at the end of the paper.
\end{abstract}

Keywords: Timeliness, Quarterly Reports, Pandemic Disease

\section{Introduction}

Financial report is very important for the stakeholders to make decisions and it comprises of balance sheet, profit and loss and cash flow statements. There are two types of financial reports that are required to be submitted by the public listed companies namely annual and interim financial reports. Annual report is published annually by referring to the companies' financial year end and interim report is published half yearly or quarterly. Malaysia Financial Reporting Standards (MFRS) defined interim as a financial report that comprehends either a complete or condensed set of financial statements for a period shorter than the entity's full financial year. Annual report is compulsory to be audited by the external auditors in order for it to be true and fair view. Interim report is optional for the organization to be audited due to shorter period of time for interim to be released. Currently, the world is facing with a pandemic disease, Covid 19 that has a great influence towards the global economic. Total lockdown and social distancing had been instructed by the Government in order to prevent the spreads of the disease and many standard operating procedures had been implemented to increase healthiness. These preventive measures caused a majority of employees to work from home and due to these limitations, will the organizations be able to submit their interims within the stipulated time set out by the Bursa Malaysia due to short time period imposed? 


\section{Literature Review}

Interim report that is published on a timely basis is very essential to the users because they are inclined to use the updated information to make decisions. According to the Listing Requirements issued by Bursa Malaysia or formerly known as Security Commission of Malaysia, paragraph/Rule 9.22, quarterly reports need to be issued within two months or 60 days after the end of each quarter of a financial year. If the companie cannot issue within the timeframe, they need to request 15 days before the timeframe expires. Otherwise, the Bursa Malaysia will not consider the application. This regulation indicates the importance for the companies to issue the quarterly reports within the stipulated timeframe. The companies need to issue quarterly reports timely because delaying to publish will provide outdated information to the financial reports' users.

There is many research on timeliness of financial reporting; the impact of audit committee on timeliness of financial reports (Syofyan et al., 2021; Ashraf et al., 2019; Zaitul and Ilona, 2019); timeliness of financial reporting in mining companies (Fauziah et al., 2020) and banking industry (Murti, 2021) and the effect of managerial abilities on timeliness of financial reports (Hashad and Eissa, 2021). Delay to issue financial reports are normally subject to unfavourable news and fear of investors to react negatively towards the companies' investments.

The pandemic disease Covid 19 or also known as coronavirus had significantly influenced the world's economy and livelihoods. Governments in many countries applied extreme policy decisions that caused negative consequences on their economy and ultimately led to global recession in 2020. The policies that were executed were impulsive, insufficient and contradicts to one another. For example, the accommodative monetary policies encouraged economic agents to engage in economic activities but the instructions by the authorities to lockdown and stay at home and social distancing policies had prevented the economic activities to operate as usual. The policy makers of many countries made challenging decision by choosing to save people from Covid 19 than to save the country's economy (Ozili and Arun 2020). This will undeniably instigate the economic recession and the recovery is anticipated to be in 2021(Açikgöz \& Günay, 2020).

There are numerous industries that were exposed to a massive economic distress due to pandemic disease such as textile industries (Kaur, 2021) because lockdown and social distances caused people to stay at home and not purchasing new textiles; aviation industry (Melas \& Melasová, 2020) due to travel restrictions; and construction industries (Alsharef et al., 2021) due to delay and inadequate workers to complete the project with standard operating procedures taking place to ensure the workers' safety. Many employees are also either being terminated due to bankruptcies of the employers or they need to work from home. Some organizations like banking and insurance necessitate to use the company's server to operate the business. Due to these limitations, can the organizations prepare the quarterly financial reports on timely basis and submit within the stipulated time period set by the Bursa Malaysia? Therefore, the present study is conducted to investigate the timeliness of quarterly financial reports of public listed 
companies in Malaysia and make comparison before the pandemic disease and the current situation where the disease still continues.

\section{Methodology}

Data were gathered from the public filings on The Bursa Malaysia Stock Exchange where the Malaysian public listed companies need to file their interims and annual reports. The industries that were included in the study were from the main market namely construction, consumer products and services, energy, financial services, health care, industrial products and services, plantation, property, real estate investment trusts, technology, telecommunication and media, transportation and logistics and utilities. 102 organizations were chosen as a sample which were randomly selected, equally from all industries.

All quarterly interims of 2019, 2020 and 2021 were selected to investigate the timeliness. However, in 2021 there were only quarter one and two that had been published. Comparison will be made across periods and industries. In 2019, the Covid 19 had just about to start at the end of the year and total lockdown was just in China and other countries remained safe at those moments. Therefore 2019 is classified as a period before the pandemic disease in Malaysia. In 2020, the year is classified as pandemic disease period. In 2021, many countries had partially or fully vaccinated their citizens and this year is classified as recovery period. There is no research that relate the timeliness to issue interims with the pandemic disease periods. Therefore, it is important for this study to be conducted in order to investigate whether the authority should reconsiderate the policies that had been imposed to the organizations to comply. The data were analyzed by using Statistical Package for Social Science (SPSS) and a descriptive statistics and paired sample T-test was performed to answer the objectives of the study.

\section{Data Analysis}

Timeliness to issue quarterly reports is very important for the public listed companies to comply. An analysis had been conducted to investigate whether the selected sample had complied with the timely submission required by Bursa Malaysia. This study found that one company from plantation industry didn't submit the quarterly reports for the fourth quarter in 2019 and 2020. This is possibly due to hectic work to publish the year end report at the same time and constraints on number of employees to work in the office due to instruction to work from home.

The number of days imposed by Bursa Malaysia to submit quarterly report is 60 days after the end of each quarter of a financial year. The number of companies that did not comply with the allowable timeframe is as per Table 1 as follows:

Table 1: Number of companies that did not comply with the allowable timeframe

\begin{tabular}{|ll|l|l|l|l|l|l|l|l|l|l|}
\hline Period(s) & & Q1 & Q2 & Q3 & Q4 & Q1 & Q2 & Q3 & Q4 & Q1 & Q2 \\
\cline { 2 - 11 } & 2019 & 2019 & 2019 & 2019 & 2020 & 2020 & 2020 & 2020 & 2021 & 2021 \\
\hline $\begin{array}{l}\text { Number } \\
\text { companies }\end{array}$ & of & 2 & 1 & 0 & 1 & 49 & 0 & 0 & 14 & 8 & 22 \\
\hline
\end{tabular}


There is a huge number of overdue submissions of quarterly reports in quarter 12020 , which is the period where the Covid 19 began. Non-compliance of submission was possibly due to extension given by Bursa Malaysia on 16 April 2020, where it had granted a month extension to issue quarterly report that was due on 31 May 2020 to 30 June 2020, following an announcement of two weeks extension of Movement Control Order (MCO) from 15 to 28 April 2020. After the extension period, the submission of quarterly reports was within the allowable timeframe for the next two quarters. However, number of delay submission had increased from 14 companies in quarter four 2020 to 22 companies in quarter two 2021. By referring to table 1, the pandemic disease had influenced the number of companies that did not comply with the allowable timeframe.

Table 2 summarize the mean of timeliness to issue quarterly reports for all and specific industries. The means indicated that all public listed companies complied with the timeframe regulation set by the Bursa Malaysia except quarter 1 2020. 48\% of the public listed companies took the opportunity to issue quarterly reports more than allowable timeframe which ultimately increase the mean to 64.8 days for quarter 1 2020. The industries that didn't comply with the allowable timeframe were construction, industrial products, energy, plantation, property, transportation and utilities. The first five industries that have low means or more timeliness to issue quarterly reports are from real estate investment trusts, telecommunication and media, health care, technology and consumer products and services. The five industries that have high means or less timeliness to issue quarterly reports are from construction, energy, property, industrial products and services and utilities.

Table 2: Mean of timeliness for all and specific industries 
Although mean of all industries complied with the allowable timeframe of 60 days for all years except quarter 1 2020, specific industries had not complied with the allowable timeframe for certain quarters during the pandemic period. In quarter four 2020, energy and property industries submission were 64.1 days and 67.1 days respectively. In quarter one 2021, there were additional two industries that did not comply with the allowable timeframe namely construction and industrial products and services. In quarter two 2021, there was an additional industry that did not comply with the timeframe which was utilities. These trends indicated that there will possibly be additional industries that cannot comply with the allowable timeframe in the future. By referring to the opportunity taken up by the organizations after extension was granted on 16 April 2020, another one industry that possibly cannot comply with the timeframe is transportation and logistics.

Table 3 indicated the minimum and maximum number of days to issue quarterly reports by the public listed companies. A company from plantation industry failed to comply with the allowable timeframe in quarter four 2019 and 2020. Excluding non-submission of quarterly reports, the minimum number of days to issue quarterly reports are 12, 14 and 16 days in year 2019, 2020 and 2021 respectively. The minimum number of days for the year are all from telecommunication

\begin{tabular}{|l|l|l|l|l|l|l|l|l|l|l|}
\hline $\begin{array}{l}\text { Type of Industry/ } \\
\text { Period(s) }\end{array}$ & $\begin{array}{l}\text { Q1 } \\
2019\end{array}$ & $\begin{array}{l}\text { Q2 } \\
2019\end{array}$ & $\begin{array}{l}\text { Q3 } \\
2019\end{array}$ & $\begin{array}{l}\text { Q4 } \\
2019\end{array}$ & $\begin{array}{l}\text { Q1 } \\
2020\end{array}$ & $\begin{array}{l}\text { Q2 } \\
2020\end{array}$ & $\begin{array}{l}\text { Q3 } \\
2020\end{array}$ & $\begin{array}{l}\text { Q4 } \\
2020\end{array}$ & $\begin{array}{l}\text { Q1 } \\
2021\end{array}$ & $\begin{array}{l}\text { Q2 } \\
2021\end{array}$ \\
\hline Construction & 57.0 & 57.8 & 57.5 & 57.4 & $\mathbf{8 6 . 9}$ & 56.9 & 55.9 & 58.6 & $\mathbf{6 2 . 0}$ & $\mathbf{7 0 . 9}$ \\
\hline $\begin{array}{l}\text { Consumer } \begin{array}{l}\text { Products and } \\
\text { services }\end{array} \\
45.1\end{array}$ & 53.1 & 48.4 & 57.0 & 55.3 & 53.4 & 49.6 & 56.0 & 46.4 & 56.9 \\
\hline $\begin{array}{l}\text { Industrial } \\
\begin{array}{l}\text { Products and } \\
\text { services }\end{array}\end{array}$ & 59.3 & 56.4 & 54.9 & 56.5 & $\mathbf{7 7 . 3}$ & 54.9 & 54.4 & 55.9 & $\mathbf{6 2 . 0}$ & $\mathbf{6 4 . 9}$ \\
\hline Energy & 57.6 & 56.8 & 57.4 & 57.5 & $\mathbf{7 0 . 9}$ & 57.5 & 57.0 & $\mathbf{6 4 . 1}$ & $\mathbf{6 1 . 6}$ & $\mathbf{7 1 . 4}$ \\
\hline Financial Services & 50.1 & 51.5 & 53.5 & 54.5 & 52.1 & 54.0 & 54.1 & 53.4 & 54.5 & 54.1 \\
\hline Health care & 51.6 & 51.4 & 53.3 & 53.5 & 57.9 & 51.3 & 46.3 & 44.9 & 44.8 & 50.3 \\
\hline Plantation & 52.5 & 47.4 & 55.1 & $\mathbf{5 6 . 1}$ & $\mathbf{6 6 . 5}$ & 52.0 & 52.6 & $\mathbf{5 9 . 9}$ & 49.9 & 51.0 \\
\hline Property & 54.3 & 50.0 & 51.0 & 57.6 & $\mathbf{7 7 . 1}$ & 55.4 & 54.9 & $\mathbf{6 7 . 1}$ & $\mathbf{6 3 . 1}$ & $\mathbf{7 1 . 6}$ \\
\hline $\begin{array}{l}\text { Real } \\
\text { investment trusts }\end{array}$ & 37.4 & 38.7 & 36.4 & 28.3 & 55.3 & 31.9 & 41.7 & 31.9 & 39.9 & 42.1 \\
\hline Technology & 53.4 & 47.6 & 52.5 & 56.0 & 58.6 & 50.6 & 49.6 & 52.4 & 44.6 & 46.0 \\
\hline $\begin{array}{l}\text { Telecommunicati } \\
\text { on and media }\end{array}$ & 48.6 & 49.7 & 45.0 & 50.3 & 49.0 & 45.6 & 45.1 & 51.1 & 47.3 & 46.9 \\
\hline $\begin{array}{l}\text { Transportation } \\
\text { and logistics }\end{array}$ & 53.6 & 47.0 & 51.1 & 52.6 & $\mathbf{6 0 . 6}$ & 50.3 & 55.5 & 56.8 & 53.3 & 59.0 \\
\hline Utilities & 55.4 & 50.0 & 53.3 & 55.1 & $\mathbf{7 1 . 5}$ & 57.0 & 55.6 & 59.9 & 57.8 & $\mathbf{6 3 . 8}$ \\
\hline All industries & 52.2 & 50.7 & 51.7 & 53.0 & $\mathbf{6 4 . 8}$ & 51.8 & 51.9 & 54.4 & 53.0 & 57.9 \\
\hline
\end{tabular}

industry. The maximum number of days to issue quarterly reports are 86, 91 and 92 in year 2019, 2020 and 2021 respectively. The maximum number of days is from telecommunication industry in 2019; numerous industries in 2020 due to extension of one month period given by Bursa 
Malaysia on 16 April 2020; and numerous industries in 2021 although no extension timeframe given by Bursa Malaysia. Figure 1 illustrates the similar minimum number of days to submit quarterly reports except plantation in quarter four 2019 and 2020 and construction in quarter one 2020.

100

Figure 1: Minimum number of days to issue quarterly reports

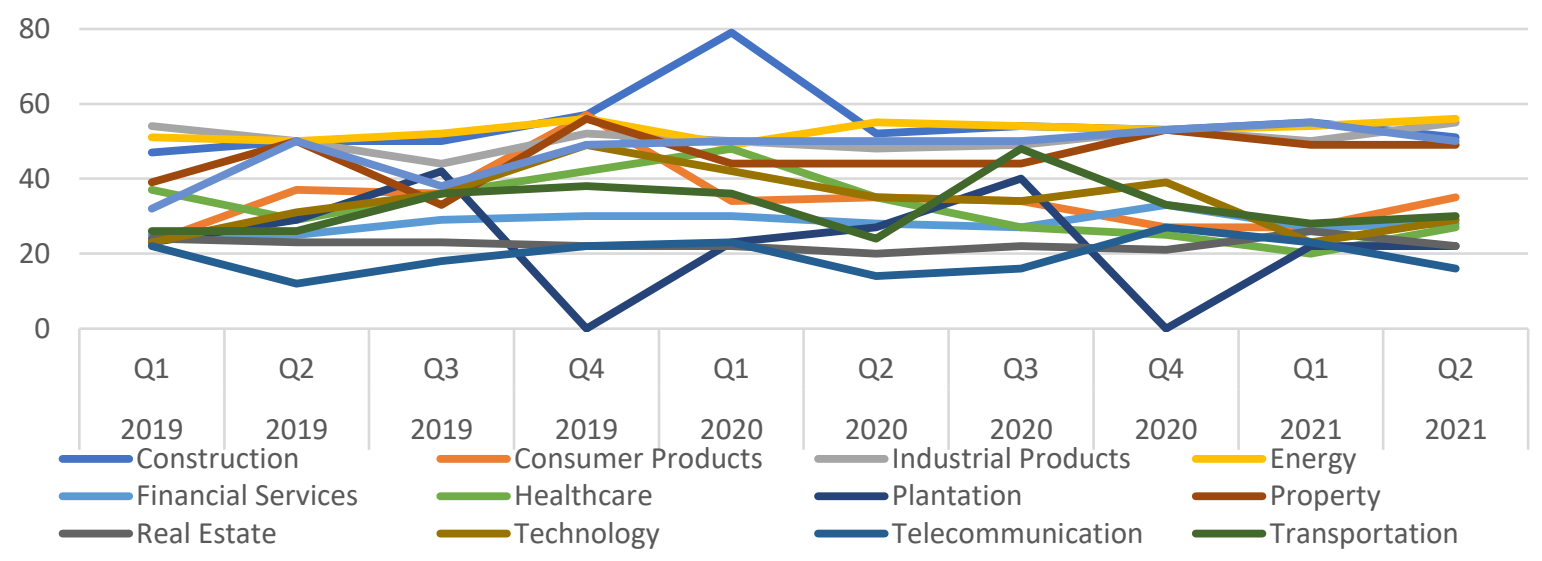

Figure 2 illustrates the maximum number of days to issue quarterly reports for various industries. In 2019, before the pandemic disease, the numbers are within the allowable timeframe of 60 days except in quarter 2 with 86 days. In 2020, during the pandemic disease, a majority took the opportunity to submit more than the allowable timeframe because extension was given by Bursa Malaysia. The trend is quite similar for the next two quarters for all industries. In quarter four 2020 and the first two quarters in 2021, during the recovery period, the graph illustrates the complexity number of days to issue the quarterly reports. Some industries managed to issue quarterly reports within the allowable timeframe, but many industries did not comply with the timeframe. This indicates the difficulties to comply with the timeframe imposed by Bursa Malaysia due to the pandemic disease. 
INTERNATIONAL JOURNAL OF ACADEMIC RESEARCH ECONOMICS AND MANAGEMENT SCIENCES Vol. 10, No. 3, 2020, E-ISSN: 2226-3624 @ 2020 HRMARS

\section{Figure 2: Maximum number of days to issue quarterly reports}

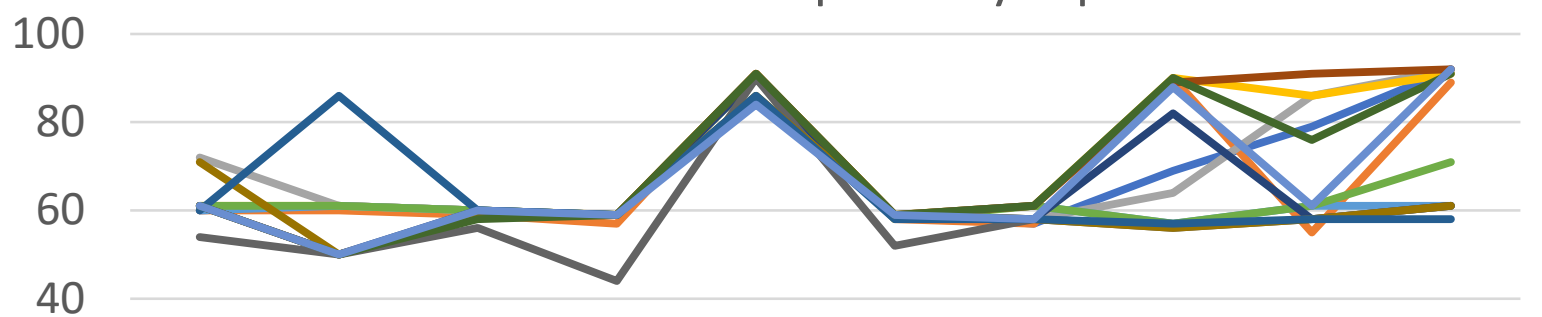

20

0

\begin{tabular}{|c|c|c|c|c|c|c|c|c|c|}
\hline Q1 & Q2 & Q3 & Q4 & Q1 & Q2 & Q3 & Q4 & Q1 & Q2 \\
\hline 2019 & 2019 & 2019 & 2019 & 2020 & 2020 & 2020 & 2020 & 2021 & 2021 \\
\hline $\begin{array}{l}\text { nstruc } \\
\text { nancial } \\
\text { al Esta } \\
\text { ilitioc }\end{array}$ & & $\begin{array}{l}C \\
\\
\end{array}$ & $\begin{array}{l}\text { umer P } \\
\text { hcare } \\
\text { hology }\end{array}$ & & $\begin{aligned} & \text { Indus } \\
& \text { Plant } \\
& \text { Telec }\end{aligned}$ & $\begin{array}{l}\text { al Produ } \\
\text { on } \\
\text { munica }\end{array}$ & 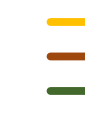 & $\begin{array}{l}\text { Energy } \\
\text { Propert } \\
\text { Transpo }\end{array}$ & \\
\hline
\end{tabular}


INTERNATIONAL JOURNAL OF ACADEMIC RESEARCH ECONOMICS AND MANAGEMENT SCIENCES

Vol. 10, No. 3, 2020, E-ISSN: 2226-3624 @ 2020 HRMARS

Table 3: The minimum and maximum number of days to submit the quarterly reports

\begin{tabular}{|c|c|c|c|c|c|c|c|c|c|c|c|c|c|c|c|c|c|c|c|c|}
\hline \multirow{3}{*}{$\begin{array}{l}\text { Type of } \\
\text { Industry/ } \\
\text { Period(s) }\end{array}$} & \multicolumn{10}{|c|}{ Minimum number of days } & \multicolumn{10}{|c|}{ Maximum number of days } \\
\hline & \multicolumn{4}{|c|}{2019} & \multicolumn{4}{|c|}{2020} & \multicolumn{2}{|c|}{2021} & \multicolumn{4}{|c|}{2019} & \multicolumn{4}{|c|}{2020} & \multicolumn{2}{|c|}{2021} \\
\hline & $\begin{array}{l}Q \\
1\end{array}$ & $\begin{array}{l}Q \\
2\end{array}$ & $\begin{array}{l}Q \\
3\end{array}$ & $\begin{array}{l}Q \\
4\end{array}$ & $\begin{array}{l}Q \\
1\end{array}$ & $\begin{array}{l}Q \\
2\end{array}$ & $\begin{array}{l}Q \\
3\end{array}$ & $\begin{array}{l}Q \\
4\end{array}$ & $\begin{array}{l}Q \\
1\end{array}$ & $\begin{array}{l}Q \\
2\end{array}$ & $\begin{array}{l}Q \\
1\end{array}$ & $\begin{array}{l}Q \\
2\end{array}$ & $\begin{array}{l}Q \\
3\end{array}$ & $\begin{array}{l}Q \\
4\end{array}$ & $\begin{array}{l}Q \\
1\end{array}$ & $\begin{array}{l}Q \\
2\end{array}$ & $\begin{array}{l}Q \\
3\end{array}$ & $\begin{array}{l}Q \\
4\end{array}$ & $\begin{array}{l}Q \\
1\end{array}$ & $\begin{array}{l}Q \\
2\end{array}$ \\
\hline $\begin{array}{l}\text { Constructio } \\
n\end{array}$ & $\begin{array}{l}4 \\
7 . \\
0\end{array}$ & $\begin{array}{l}5 \\
0 . \\
0\end{array}$ & $\begin{array}{l}5 \\
0 . \\
0\end{array}$ & $\begin{array}{l}5 \\
7 . \\
0\end{array}$ & $\begin{array}{l}7 \\
9 . \\
0\end{array}$ & $\begin{array}{l}5 \\
2 . \\
0\end{array}$ & $\begin{array}{l}5 \\
4 . \\
0\end{array}$ & $\begin{array}{l}5 \\
3 . \\
0\end{array}$ & $\begin{array}{l}5 \\
5 . \\
0\end{array}$ & $\begin{array}{l}5 \\
1 . \\
0\end{array}$ & $\begin{array}{l}6 \\
0 . \\
0\end{array}$ & $\begin{array}{l}6 \\
1 . \\
0\end{array}$ & $\begin{array}{l}6 \\
0 . \\
0\end{array}$ & $\begin{array}{l}5 \\
9 . \\
0\end{array}$ & $\begin{array}{l}9 \\
1 . \\
0\end{array}$ & $\begin{array}{l}5 \\
9 . \\
0\end{array}$ & $\begin{array}{l}5 \\
7 . \\
0\end{array}$ & $\begin{array}{l}6 \\
9 . \\
0\end{array}$ & $\begin{array}{l}7 \\
9 . \\
0\end{array}$ & $\begin{array}{l}9 \\
2 . \\
0\end{array}$ \\
\hline $\begin{array}{l}\text { Consumer } \\
\text { Products }\end{array}$ & $\begin{array}{l}2 \\
3 . \\
0\end{array}$ & $\begin{array}{l}3 \\
7 . \\
0\end{array}$ & $\begin{array}{l}3 \\
6 . \\
0\end{array}$ & $\begin{array}{l}5 \\
7 . \\
0\end{array}$ & $\begin{array}{l}3 \\
4 . \\
0\end{array}$ & $\begin{array}{l}3 \\
5 . \\
0\end{array}$ & $\begin{array}{l}3 \\
4 . \\
0\end{array}$ & $\begin{array}{l}2 \\
7 . \\
0\end{array}$ & $\begin{array}{l}2 \\
7 . \\
0\end{array}$ & $\begin{array}{l}3 \\
5 . \\
0\end{array}$ & $\begin{array}{l}6 \\
0 . \\
0\end{array}$ & $\begin{array}{l}6 \\
0 . \\
0\end{array}$ & $\begin{array}{l}5 \\
9 . \\
0\end{array}$ & $\begin{array}{l}5 \\
7 . \\
0\end{array}$ & $\begin{array}{l}9 \\
1 . \\
0\end{array}$ & $\begin{array}{l}5 \\
8 . \\
0\end{array}$ & $\begin{array}{l}5 \\
7 . \\
0\end{array}$ & $\begin{array}{l}9 \\
0 . \\
0\end{array}$ & $\begin{array}{l}5 \\
5 . \\
0\end{array}$ & $\begin{array}{l}8 \\
9 . \\
0\end{array}$ \\
\hline $\begin{array}{l}\text { Industrial } \\
\text { Products }\end{array}$ & $\begin{array}{l}5 \\
4 . \\
0\end{array}$ & $\begin{array}{l}5 \\
0 . \\
0\end{array}$ & $\begin{array}{l}4 \\
4 . \\
0\end{array}$ & $\begin{array}{l}5 \\
2 . \\
0\end{array}$ & $\begin{array}{l}5 \\
0 . \\
0\end{array}$ & $\begin{array}{l}4 \\
8 . \\
0\end{array}$ & $\begin{array}{l}4 \\
9 . \\
0 .\end{array}$ & $\begin{array}{l}5 \\
3 . \\
0\end{array}$ & $\begin{array}{l}5 \\
0 .\end{array}$ & $\begin{array}{l}5 \\
5 . \\
0\end{array}$ & $\begin{array}{l}7 \\
2 . \\
0\end{array}$ & $\begin{array}{l}6 \\
1 . \\
0\end{array}$ & $\begin{array}{l}6 \\
0 . \\
0\end{array}$ & $\begin{array}{l}5 \\
9 . \\
0\end{array}$ & $\begin{array}{l}9 \\
1 . \\
0\end{array}$ & $\begin{array}{l}5 \\
9 . \\
0\end{array}$ & $\begin{array}{l}5 \\
8 . \\
0\end{array}$ & $\begin{array}{l}6 \\
4 . \\
0\end{array}$ & $\begin{array}{l}8 \\
6 . \\
0\end{array}$ & $\begin{array}{l}9 \\
2 . \\
0\end{array}$ \\
\hline Energy & $\begin{array}{l}5 \\
1 . \\
0\end{array}$ & $\begin{array}{l}5 \\
0 . \\
0\end{array}$ & $\begin{array}{l}5 \\
2 . \\
0\end{array}$ & $\begin{array}{l}5 \\
6 . \\
0\end{array}$ & $\begin{array}{l}4 \\
9 . \\
0\end{array}$ & $\begin{array}{l}5 \\
5 . \\
0\end{array}$ & $\begin{array}{l}5 \\
4 . \\
0\end{array}$ & $\begin{array}{l}5 \\
3 . \\
0\end{array}$ & $\begin{array}{l}5 \\
4 . \\
0\end{array}$ & $\begin{array}{l}5 \\
6 . \\
0\end{array}$ & $\begin{array}{l}6 \\
1 . \\
0\end{array}$ & $\begin{array}{l}6 \\
1 . \\
0\end{array}$ & $\begin{array}{l}6 \\
0 . \\
0\end{array}$ & $\begin{array}{l}5 \\
9 . \\
0\end{array}$ & $\begin{array}{l}9 \\
1 . \\
0\end{array}$ & $\begin{array}{l}5 \\
9 . \\
0\end{array}$ & $\begin{array}{l}6 \\
1 . \\
0\end{array}$ & $\begin{array}{l}9 \\
0 . \\
0\end{array}$ & $\begin{array}{l}8 \\
6 . \\
0\end{array}$ & $\begin{array}{l}9 \\
1 . \\
0\end{array}$ \\
\hline $\begin{array}{l}\text { Financial } \\
\text { Services }\end{array}$ & $\begin{array}{l}2 \\
5 . \\
0\end{array}$ & $\begin{array}{l}2 \\
5 . \\
0\end{array}$ & $\begin{array}{l}2 \\
9 . \\
0\end{array}$ & $\begin{array}{l}3 \\
0 . \\
0\end{array}$ & $\begin{array}{l}3 \\
0 . \\
0\end{array}$ & $\begin{array}{l}2 \\
8 . \\
0\end{array}$ & $\begin{array}{l}2 \\
7 . \\
0\end{array}$ & $\begin{array}{l}3 \\
3 . \\
0\end{array}$ & $\begin{array}{l}2 \\
7 . \\
0\end{array}$ & $\begin{array}{l}2 \\
8 . \\
0\end{array}$ & $\begin{array}{l}6 \\
0 . \\
0\end{array}$ & $\begin{array}{l}6 \\
1 . \\
0\end{array}$ & $\begin{array}{l}6 \\
0 . \\
0\end{array}$ & $\begin{array}{l}5 \\
9 . \\
0 .\end{array}$ & $\begin{array}{l}8 \\
6 . \\
0\end{array}$ & $\begin{array}{l}5 \\
9 . \\
0\end{array}$ & $\begin{array}{l}6 \\
1 . \\
0\end{array}$ & $\begin{array}{l}5 \\
7 . \\
0\end{array}$ & $\begin{array}{l}6 \\
1 . \\
0\end{array}$ & $\begin{array}{l}6 \\
1 . \\
0\end{array}$ \\
\hline Healthcare & $\begin{array}{l}3 \\
7 . \\
0\end{array}$ & $\begin{array}{l}2 \\
9 . \\
0\end{array}$ & $\begin{array}{l}3 \\
6 . \\
0\end{array}$ & $\begin{array}{l}4 \\
2 . \\
0\end{array}$ & $\begin{array}{l}4 \\
8 . \\
0\end{array}$ & $\begin{array}{l}3 \\
5 . \\
0\end{array}$ & $\begin{array}{l}2 \\
7 . \\
0\end{array}$ & $\begin{array}{l}2 \\
5 . \\
0\end{array}$ & $\begin{array}{l}2 \\
0 .\end{array}$ & $\begin{array}{l}2 \\
7 . \\
0\end{array}$ & $\begin{array}{l}6 \\
1 . \\
0\end{array}$ & $\begin{array}{l}6 \\
1 . \\
0\end{array}$ & $\begin{array}{l}6 \\
0 . \\
0\end{array}$ & $\begin{array}{l}5 \\
9 . \\
0\end{array}$ & $\begin{array}{l}9 \\
0 . \\
0\end{array}$ & $\begin{array}{l}5 \\
8 . \\
0\end{array}$ & $\begin{array}{l}6 \\
1 . \\
0\end{array}$ & $\begin{array}{l}5 \\
7 . \\
0\end{array}$ & $\begin{array}{l}6 \\
1 . \\
0\end{array}$ & $\begin{array}{l}7 \\
1 . \\
0\end{array}$ \\
\hline Plantation & $\begin{array}{l}2 \\
3 . \\
0\end{array}$ & $\begin{array}{l}2 \\
9 . \\
0\end{array}$ & $\begin{array}{l}4 \\
2 . \\
0\end{array}$ & $\begin{array}{l}0 . \\
0\end{array}$ & $\begin{array}{l}2 \\
3 . \\
0\end{array}$ & $\begin{array}{l}2 \\
7 . \\
0\end{array}$ & $\begin{array}{l}4 \\
0 . \\
0\end{array}$ & $\begin{array}{l}0 . \\
0\end{array}$ & $\begin{array}{l}2 \\
2 . \\
0\end{array}$ & $\begin{array}{l}2 \\
2 . \\
0 .\end{array}$ & $\begin{array}{l}6 \\
1 . \\
0\end{array}$ & $\begin{array}{l}5 \\
0 . \\
0 .\end{array}$ & $\begin{array}{l}6 \\
0 . \\
0 .\end{array}$ & $\begin{array}{l}5 \\
9 . \\
0 .\end{array}$ & $\begin{array}{l}9 \\
0 . \\
0\end{array}$ & $\begin{array}{l}5 \\
9 . \\
0\end{array}$ & $\begin{array}{l}5 \\
8 . \\
0\end{array}$ & $\begin{array}{l}8 \\
2 . \\
0\end{array}$ & $\begin{array}{l}5 \\
8 . \\
0\end{array}$ & $\begin{array}{l}6 \\
1 . \\
0\end{array}$ \\
\hline Property & $\begin{array}{l}3 \\
9 . \\
0\end{array}$ & $\begin{array}{l}5 \\
0 . \\
0\end{array}$ & $\begin{array}{l}3 \\
3 . \\
0\end{array}$ & $\begin{array}{l}5 \\
6 . \\
0\end{array}$ & $\begin{array}{l}4 \\
4 . \\
0\end{array}$ & $\begin{array}{l}4 \\
4 . \\
0\end{array}$ & $\begin{array}{l}4 \\
4 . \\
0\end{array}$ & $\begin{array}{l}5 \\
3 . \\
0\end{array}$ & $\begin{array}{l}4 \\
9 . \\
0\end{array}$ & $\begin{array}{l}4 \\
9 . \\
0\end{array}$ & $\begin{array}{l}6 \\
1 . \\
0\end{array}$ & $\begin{array}{l}5 \\
0 . \\
0 .\end{array}$ & $\begin{array}{l}5 \\
9 . \\
0\end{array}$ & $\begin{array}{l}5 \\
9 . \\
0\end{array}$ & $\begin{array}{l}9 \\
1 . \\
0\end{array}$ & $\begin{array}{l}5 \\
9 . \\
0\end{array}$ & $\begin{array}{l}6 \\
1 . \\
0\end{array}$ & $\begin{array}{l}8 \\
9 . \\
0\end{array}$ & $\begin{array}{l}9 \\
1 . \\
0\end{array}$ & $\begin{array}{l}9 \\
2 . \\
0\end{array}$ \\
\hline Real Estate & $\begin{array}{l}2 \\
4 . \\
0\end{array}$ & $\begin{array}{l}2 \\
3 . \\
0\end{array}$ & $\begin{array}{l}2 \\
3 . \\
0\end{array}$ & $\begin{array}{l}2 \\
2 . \\
0\end{array}$ & $\begin{array}{l}2 \\
2 . \\
0\end{array}$ & $\begin{array}{l}2 \\
0 . \\
0\end{array}$ & $\begin{array}{l}2 \\
2 . \\
0\end{array}$ & $\begin{array}{l}2 \\
1 . \\
0\end{array}$ & $\begin{array}{l}2 \\
6 .\end{array}$ & $\begin{array}{l}2 \\
2 . \\
0\end{array}$ & $\begin{array}{l}5 \\
4 . \\
0\end{array}$ & $\begin{array}{l}5 \\
0 . \\
0 .\end{array}$ & $\begin{array}{l}5 \\
6 . \\
0\end{array}$ & $\begin{array}{l}4 \\
4 . \\
0\end{array}$ & $\begin{array}{l}9 \\
0 . \\
0\end{array}$ & $\begin{array}{l}5 \\
2 . \\
0\end{array}$ & $\begin{array}{l}5 \\
8 . \\
0\end{array}$ & $\begin{array}{l}5 \\
6 . \\
0\end{array}$ & $\begin{array}{l}5 \\
8 . \\
0\end{array}$ & $\begin{array}{l}6 \\
1 . \\
0\end{array}$ \\
\hline Technology & $\begin{array}{l}2 \\
3 . \\
0\end{array}$ & $\begin{array}{l}3 \\
1 . \\
0\end{array}$ & $\begin{array}{l}3 \\
6 . \\
0\end{array}$ & $\begin{array}{l}4 \\
9 . \\
0\end{array}$ & $\begin{array}{l}4 \\
2 . \\
0\end{array}$ & $\begin{array}{l}3 \\
5 . \\
0\end{array}$ & $\begin{array}{l}3 \\
4 . \\
0\end{array}$ & $\begin{array}{l}3 \\
9 . \\
0\end{array}$ & $\begin{array}{l}2 \\
3 . \\
0\end{array}$ & $\begin{array}{l}2 \\
9 . \\
0\end{array}$ & $\begin{array}{l}7 \\
1 . \\
0\end{array}$ & $\begin{array}{l}5 \\
0 . \\
0 .\end{array}$ & $\begin{array}{l}5 \\
9 . \\
0 .\end{array}$ & $\begin{array}{l}5 \\
9 . \\
0 .\end{array}$ & $\begin{array}{l}8 \\
6 . \\
0\end{array}$ & $\begin{array}{l}5 \\
9 . \\
0\end{array}$ & $\begin{array}{l}5 \\
8 . \\
0\end{array}$ & $\begin{array}{l}5 \\
6 . \\
0 .\end{array}$ & $\begin{array}{l}5 \\
8 . \\
0\end{array}$ & $\begin{array}{l}6 \\
1 . \\
0\end{array}$ \\
\hline $\begin{array}{l}\text { Telecommu } \\
\text { nication }\end{array}$ & $\begin{array}{l}2 \\
2 . \\
0\end{array}$ & $\begin{array}{l}1 \\
2 . \\
0\end{array}$ & $\begin{array}{l}1 \\
8 . \\
0\end{array}$ & $\begin{array}{l}2 \\
2 . \\
0\end{array}$ & $\begin{array}{l}2 \\
3 . \\
0\end{array}$ & $\begin{array}{l}1 \\
4 . \\
0\end{array}$ & $\begin{array}{l}1 \\
6 . \\
0\end{array}$ & $\begin{array}{l}2 \\
7 . \\
0\end{array}$ & $\begin{array}{l}2 \\
3 . \\
0\end{array}$ & $\begin{array}{l}1 \\
6 . \\
0\end{array}$ & $\begin{array}{l}6 \\
0 . \\
0\end{array}$ & $\begin{array}{l}8 \\
6 . \\
0\end{array}$ & $\begin{array}{l}6 \\
0 . \\
0\end{array}$ & $\begin{array}{l}5 \\
9 . \\
0\end{array}$ & $\begin{array}{l}8 \\
6 . \\
0\end{array}$ & $\begin{array}{l}5 \\
8 . \\
0\end{array}$ & $\begin{array}{l}5 \\
8 . \\
0\end{array}$ & $\begin{array}{l}5 \\
7 . \\
0\end{array}$ & $\begin{array}{l}5 \\
8 . \\
0\end{array}$ & $\begin{array}{l}5 \\
8 . \\
0\end{array}$ \\
\hline
\end{tabular}


INTERNATIONAL JOURNAL OF ACADEMIC RESEARCH ECONOMICS AND MANAGEMENT SCIENCES

Vol. 10 , No. 3, 2020, E-ISSN: $2226-3624$ ㄷ 2020 HRMARS

\begin{tabular}{|l|l|l|l|l|l|l|l|l|l|l|l|l|l|l|l|l|l|l|l|l|}
\hline Transportati & 2 & 2 & 3 & 3 & 3 & 2 & 4 & 3 & 2 & 3 & 6 & 5 & 5 & 5 & 9 & 5 & 6 & 9 & 7 & 9 \\
on & 6. & 6. & 6. & 8. & 6. & 4. & 8. & 3. & 8. & 0. & 1. & 0. & 8. & 9. & 1. & 9. & 1. & 0. & 6. & 1. \\
& 0 & 0 & 0 & 0 & 0 & 0 & 0 & 0 & 0 & 0 & 0 & 0 & 0 & 0 & 0 & 0 & 0 & 0 & 0 & 0 \\
\hline Utilities & 3 & 5 & 3 & 4 & 5 & 5 & 5 & 5 & 5 & 5 & 6 & 5 & 6 & 5 & 8 & 5 & 5 & 8 & 6 & 9 \\
& 2. & 0. & 8. & 9. & 0. & 0. & 0. & 3. & 5. & 0. & 1. & 0. & 0. & 9. & 4. & 9. & 8. & 8. & 1. & 2. \\
& 0 & 0 & 0 & 0 & 0 & 0 & 0 & 0 & 0 & 0 & 0 & 0 & 0 & 0 & 0 & 0 & 0 & 0 & 0 & 0 \\
\hline All & 2 & 1 & 1 & 2 & 2 & 1 & 1 & 2 & 2 & 1 & 7 & 8 & 6 & 5 & 9 & 5 & 6 & 9 & 9 & 9 \\
industries & 2 & 2 & 8 & 2 & 2 & 4 & 6 & 1 & 0 & 6 & 2 & 6 & 0 & 9 & 1 & 9 & 1 & 0 & 1 & 2 \\
\hline
\end{tabular}

Note: 0 - non submission of quarterly reports

A paired sample T-test was conducted to compare the timeliness to submit quarterly reports across quarters and years and also industries. Table 4 summarize the paired sample statistics for all quarters. There was a statistically significant difference of timeliness in Pair 1,5 and 6 , proven by sig. ( 2 tailed) which is less than 0.05 and t values of $-7.623,7.23$ and -4.614 respectively. Comparing the mean values of pair 1 , the mean before pandemic disease began is lower than after the pandemic had occurred, which indicates that the pandemic disease has an influence on the timeliness for the companies to issue timely reports. Comparing the mean of pair 5 , the mean of timeliness during the recovery period is much better than the period where the pandemic disease had started because extension of one month period was given by Bursa Malaysia during the pandemic disease. Therefore, the difference only occurs during quarter one and not the remaining quarters when comparison is made before and after the pandemic disease had started. Significance also occurred for pair 6 quarter 2 for pandemic period and recovery period but at lower mean differences.

Table 4: Paired Samples Test

\begin{tabular}{|l|l|l|l|l|l|}
\hline \multicolumn{2}{|l|}{ Pair/Period } & Mean & \multicolumn{1}{|l|}{$\begin{array}{l}\text { Mean } \\
\text { difference }\end{array}$} & t- values & Sig. (2-tailed) \\
\hline Pair 1 & Q12019 & 52.17 & -12.608 & -7.623 & $\mathbf{0 . 0 0 *}$ \\
\cline { 2 - 6 } & Q12020 & 64.77 & & & \\
\hline Pair 2 & Q22019 & 50.69 & -1.147 & -1.399 & 0.17 \\
& Q22020 & 51.83 & & & \\
\hline \multirow{2}{*}{ Pair 3 } & Q32019 & 51.70 & -.186 & -0.27 & 0.79 \\
\cline { 2 - 6 } & Q32020 & 51.88 & & & \\
\hline Pair 4 & Q42019 & 52.99 & -1.441 & -1.334 & 0.19 \\
\cline { 2 - 6 } & Q42020 & 54.43 & & & \\
\hline Pair 5 & Q12020 & 64.77 & 11.745 & 7.23 & $\mathbf{0 . 0 0 *}$ \\
\hline & Q12021 & 53.03 & & & \\
\hline Pair 6 & Q22020 & 51.83 & -6.020 & -4.614 & $\mathbf{0 . 0 0 *}$ \\
\cline { 2 - 6 } & Q22021 & 57.85 & & & \\
\hline
\end{tabular}

Eta squared is calculated in order to identify the magnitude of the intervention's effect. According to Cohen (1988), the eta squared which is equal to 0.01 is considered small effect, 0.06 is moderate effect and higher than 0.14 is large effect. This study found that eta squared for pair 1 , 
INTERNATIONAL JOURNAL OF ACADEMIC RESEARCH ECONOMICS AND MANAGEMENT SCIENCES Vol. 10 , No. 3, 2020, E-ISSN: $2226-3624$ @ 2020 HRMARS

5 and 6 are $0.37,0.34$ and 0.17 respectively. These indicates that the timeliness differences between the paired quarters are high.

Table 5: Paired Samples Test (Industries)

\begin{tabular}{|c|c|c|c|c|c|c|c|c|c|c|c|c|c|}
\hline \multirow{2}{*}{\multicolumn{2}{|c|}{ Type of Industries/ Period(s) }} & \multicolumn{2}{|c|}{ Pair 1} & \multicolumn{2}{|c|}{ Pair 2} & \multicolumn{2}{|c|}{ Pair 3} & \multicolumn{2}{|c|}{ Pair 4} & \multicolumn{2}{|c|}{ Pair 5} & \multicolumn{2}{|c|}{ Pair 6} \\
\hline & & Q12019 & Q12020 & Q22019 & Q22020 & Q32019 & Q32020 & Q42019 & Q42020 & Q12020 & Q12021 & Q22020 & Q22021 \\
\hline \multirow{4}{*}{ Construction } & mean & 57.00 & 86.88 & 57.75 & 56.88 & 57.50 & 55.88 & 57.38 & 58.63 & 86.88 & 62.00 & 56.88 & 70.88 \\
\hline & Mean Diff & -29.88 & & 0.88 & & 1.63 & & -1.25 & & 24.88 & & -14.00 & \\
\hline & t- values & -12.80 & & 0.73 & & 1.76 & & -0.57 & & 7.09 & & -2.49 & \\
\hline & \begin{tabular}{|l} 
Sig. (2-tailed) \\
\end{tabular} & 0.00 & & 0.49 & & 0.12 & & 0.59 & & 0.00 & & 0.04 & \\
\hline \multirow{4}{*}{ Consumer Products } & mean & 45.13 & 55.25 & 53.13 & 53.38 & 48.38 & 49.63 & 57.00 & 56.00 & 55.25 & 46.38 & 53.38 & 56.88 \\
\hline & Mean Diff & -10.13 & & -0.25 & & -1.25 & & 1.00 & & 8.88 & & -3.50 & \\
\hline & t- values & \begin{tabular}{|c|}
-1.42 \\
\end{tabular} & & -0.25 & & -0.48 & & 0.17 & & 1.56 & & -0.86 & \\
\hline & Sig. (2-tailed) & 0.20 & & 0.81 & & 0.65 & & 0.87 & & 0.16 & & 0.42 & \\
\hline \multirow{4}{*}{ Industrial Products } & mean & 59.25 & 77.25 & 56.38 & 54.88 & 54.88 & 54.38 & 56.50 & 55.88 & 77.25 & 62.00 & 54.88 & 64.88 \\
\hline & Mean Diff & -18.00 & & 1.50 & & 0.50 & & 0.63 & & 15.25 & & -10.00 & \\
\hline & t- values & -3.57 & & 1.03 & & 0.31 & & 0.54 & & 2.92 & & -2.42 & \\
\hline & \begin{tabular}{|l} 
Sig. (2-tailed) \\
\end{tabular} & 0.01 & & 0.34 & & 0.76 & & 0.60 & & 0.02 & & 0.05 & \\
\hline \multirow{4}{*}{ Energy } & mean & 57.63 & 70.88 & 56.75 & 57.50 & 57.38 & 57.00 & 57.50 & 64.13 & 70.88 & 61.63 & 57.50 & 71.38 \\
\hline & Mean Diff & -13.25 & & -0.75 & & 0.38 & & -6.63 & & 9.25 & & \begin{tabular}{|c|}
-13.88 \\
\end{tabular} & \\
\hline & t- values & -2.17 & & -0.48 & & 0.36 & & -1.30 & & 1.08 & & -2.42 & \\
\hline & Sig. (2-tailed) & 0.07 & & 0.64 & & 0.73 & & 0.24 & & 0.31 & & 0.05 & \\
\hline \multirow{4}{*}{ Financial Services } & mean & 50.13 & 52.13 & 51.50 & 54.00 & 53.50 & 54.13 & 54.50 & 53.38 & 52.13 & 54.50 & 54.00 & 54.13 \\
\hline & Mean Diff & -2.00 & & -2.50 & & \begin{tabular}{|c|}
-0.63 \\
\end{tabular} & & 1.13 & & \begin{tabular}{|c|}
-2.38 \\
\end{tabular} & & \begin{tabular}{|c|}
-0.13 \\
\end{tabular} & \\
\hline & $\mathrm{t}$ - values & -0.33 & & -0.60 & & -0.63 & & 1.69 & & -0.48 & & -0.17 & \\
\hline & \begin{tabular}{|l} 
Sig. (2-tailed) \\
(2)
\end{tabular} & 0.75 & & 0.57 & & 0.55 & & 0.14 & & 0.65 & & 0.87 & \\
\hline \multirow{4}{*}{ Healthcare } & mean & 51.63 & 57.88 & 51.38 & 51.25 & 53.25 & 46.25 & 53.50 & 44.88 & 57.88 & 44.75 & 51.25 & 50.25 \\
\hline & Mean Diff & \begin{tabular}{|c|}
-6.25 \\
\end{tabular} & & 0.13 & & 7.00 & & 8.63 & & 13.13 & & 1.00 & \\
\hline & t- values & -1.53 & & 0.03 & & 1.57 & & 2.27 & & 2.93 & & 0.18 & \\
\hline & Sig. (2-tailed) & 0.17 & & 0.98 & & 0.16 & & 0.06 & & 0.02 & & 0.86 & \\
\hline \multirow{4}{*}{ Plantation } & mean & 52.50 & 66.50 & 47.38 & 52.00 & 55.13 & 52.63 & 49.13 & 52.38 & 66.50 & 49.88 & 52.00 & 51.00 \\
\hline & Mean Diff & -14.00 & & -4.63 & & 2.50 & & -3.25 & & 16.63 & & 1.00 & \\
\hline & t- values & -2.34 & & -2.93 & & 2.76 & & -0.85 & & 3.25 & & 0.63 & \\
\hline & \begin{tabular}{|l} 
Sig. (2-tailed) \\
\end{tabular} & 0.05 & & 0.02 & & 0.03 & & 0.42 & & 0.01 & & 0.55 & \\
\hline \multirow{4}{*}{ Property } & mean & 54.25 & 77.13 & 50.00 & 55.38 & 51.00 & 54.88 & 57.63 & 67.13 & 77.13 & 63.13 & 55.38 & 71.63 \\
\hline & Mean Diff & \begin{tabular}{|c|}
-22.88 \\
\end{tabular} & & -5.38 & & \begin{tabular}{|c|}
-3.88 \\
\end{tabular} & & -9.50 & & 14.00 & & -16.25 & \\
\hline & t- values & -3.95 & & -2.79 & & -1.05 & & -1.65 & & 1.93 & & -2.66 & \\
\hline & \begin{tabular}{|l|} 
Sig. (2-tailed) \\
\end{tabular} & 0.01 & & 0.03 & & 0.33 & & 0.14 & & 0.10 & & 0.03 & \\
\hline & mean & 37.43 & 55.29 & 38.71 & 31.86 & 36.43 & 41.71 & 28.29 & 31.86 & 55.29 & 39.86 & 31.86 & 42.14 \\
\hline Donl Fototo & Mean Diff & \begin{tabular}{|c|}
-17.86 \\
\end{tabular} & & 6.86 & & -5.29 & & $\mid-3.57$ & & 15.43 & & -10.29 & \\
\hline neal Lstale & $t$ - values & -2.29 & & 2.42 & & -1.94 & & -1.60 & & 1.98 & & -3.03 & \\
\hline & Sig. (2-tailed) & 0.06 & & 0.05 & & 0.10 & & 0.16 & & 0.10 & & 0.02 & \\
\hline & mean & 53.38 & 58.63 & 47.63 & 50.63 & 52.50 & 49.63 & 56.00 & 52.38 & 58.63 & 44.63 & 50.63 & 46.00 \\
\hline Tonhnolnay & Mean Diff & -5.25 & & -3.00 & & 2.88 & & 3.63 & & 14.00 & & 4.63 & \\
\hline 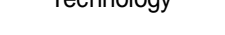 & $t$ - values & -1.09 & & -1.27 & & 2.04 & & 1.41 & & 2.36 & & 1.42 & \\
\hline & Sig. (2-tailed) & 0.31 & & 0.24 & & 0.08 & & 0.20 & & 0.05 & & 0.20 & \\
\hline & mean & 48.57 & 49.00 & 49.71 & 45.57 & 45.00 & 45.14 & 50.29 & 51.14 & 49.00 & 47.29 & 45.57 & 46.86 \\
\hline Trlo rmmuniontis & Mean Diff & -0.43 & & 4.14 & & -0.14 & & -0.86 & & 1.71 & & -1.29 & \\
\hline 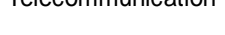 & t- values & -0.09 & & 0.65 & & -0.11 & & -0.43 & & 0.38 & & -0.82 & \\
\hline & Sig. (2-tailed) & 0.93 & & 0.54 & & 0.92 & & 0.68 & & 0.72 & & 0.44 & \\
\hline & mean & 53.63 & 60.63 & 47.00 & 50.25 & 51.13 & 55.50 & 52.63 & 56.75 & 60.63 & 53.25 & 50.25 & 59.00 \\
\hline Trononoutotion & Mean Diff & -7.00 & & -3.25 & & -4.38 & & -4.13 & & 7.38 & & -8.75 & \\
\hline 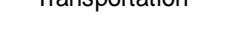 & t- values & -1.09 & & -1.73 & & -1.67 & & -0.94 & & 1.68 & & -1.67 & \\
\hline & Sig. (2-tailed) & 0.31 & & 0.13 & & 0.14 & & 0.38 & & 0.14 & & 0.14 & \\
\hline & mean & 55.38 & 71.50 & 50.00 & 57.00 & 53.25 & 55.63 & 55.13 & 59.88 & 71.50 & 57.75 & 57.00 & 63.75 \\
\hline Utiliting & Mean Diff & \begin{tabular}{|c|}
-16.13 \\
\end{tabular} & & -7.00 & & -2.38 & & -4.75 & & 13.75 & & -6.75 & \\
\hline Uimilies & t- values & -4.10 & & -6.45 & & -0.95 & & -1.25 & & 2.94 & & -1.57 & \\
\hline & Sig. (2-tailed) & 0.01 & & 0.00 & & 0.37 & & 0.25 & & 0.02 & & 0.16 & \\
\hline
\end{tabular}


Table 5 summarize the paired sample T-test for all industries. The difference of timeliness varies across industries. A quite number of industries have significant difference in timeliness to issue quarterly reports in pair 1, 2, 5 and 6 (i.e. quarter one and two for all years, 2019, 2020 and 2021). There is no significant differences of timeliness in pair 3 and 4 except plantation in quarter three. Four industries that had no difference in timeliness to issue quarterly reports were consumer products, financial services, telecommunication and media and transportation and logistics. The industries with one time difference of timeliness are energy, healthcare, technology. The industries with frequent difference of timeliness to issue quarterly reports are construction, industrial products, plantation, and utilities.

\section{Conclusion}

The main objective of this research is to find the timeliness of Malaysian public listed companies to issue quarterly reports and make comparison for three periods namely before pandemic, during pandemic and recovery period of pandemic. Additionally, this study also investigated the timeliness for all industries across all quarters. The findings suggested that all public listed companies tried hard to comply with the allowable timeframe set by Bursa Malaysia. The timeframe before the pandemic is acceptable with only a few companies that did not comply. During the pandemic disease, there were many companies that did not comply especially during quarter one $\mathbf{2 0 2 0}$ where a month extension was granted to the companies to issue quarterly reports and during last quarter of 2020. In recovery period, mean of timeliness exceeding the allowable timeframe for some companies in certain industries. Nevertheless four industries had fully complied with the allowable timeframe across quarters and years which are consumer products and services, real estate investment trusts, technology and telecommunication and media.

These findings provide implications to the policy makers where the companies may have problems with preparation of quarterly reports to be issued especially during the pandemic disease and recovery periods, possibly due to the instruction to work from home and economic recession. The policy makers should re-consider the allowable timeframe to issue financial reports because the companies may experience many challenges that should be determined in future research. 
INTERNATIONAL JOURNAL OF ACADEMIC RESEARCH ECONOMICS AND MANAGEMENT SCIENCES

Vol. 10 , No. 3, 2020, E-ISSN: 2226-3624 C 2020 HRMARS

\section{References}

Açikgöz, Ö., \& Günay, A. (2020). The early impact of the Covid-19 pandemic on the global and Turkish economy. Turkish Journal of Medical Sciences, 50(SI-1). https://doi.org/10.3906/sag-2004-6

Alsharef, A., Banerjee, S., Uddin, S. M. J., Albert, A., \& Jaselskis, E. (2021). Early impacts of the COVID-19 pandemic on the United States construction industry. International Journal of Environmental Research and Public Health, 18(4). https://doi.org/10.3390/ijerph18041559

Hashad, T. M., \& Eissa, A. M. (2021). The Effect of Managerial Ability on Financial Reporting Timeliness: Egypt Evidence. Journal of Accounting Finance and Auditing Studies (JAFAS), 7(3). https://doi.org/10.32602/jafas.2021.020

Kaur, K. (2021). The Early Impact of COVID-19 on Textile Industry: An Empirical Analysis. Management and Labour Studies, 46(3). https://doi.org/10.1177/0258042X21991018

Melas, D., \& Melasová, K. (2020). The Early Impact of Covid-19 Pandemic on the Aviation Industry. Acta Avionica Journal. https://doi.org/10.35116/aa.2020. 0005

Murti, W. (2021). Timeliness of corporate annual financial reporting in Indonesian banking industry. Accounting, 7(3). https://doi.org/10.5267/j.ac. 2021.1.003

Ozili, P. K., \& Arun, T. (2020). Spillover of COVID-19: Impact on the Global Economy. SSRN Electronic Journal. https://doi.org/10.2139/ssrn.3562570

Syofyan, E., Septiari, D., Dwita, S., \& Rahmi, M. (2021). The characteristics of the audit committee affecting timeliness of the audit report in Indonesia. Cogent Business and Management, 8(1). https://doi.org/10.1080/23311975.2021. 1935183

Zaitul, \& Ilona, D. (2019). Gender in audit committee and financial reporting timeliness: The case of unique continental European model. International Journal of Recent Technology and Engineering, 8(2S9). https://doi.org/10.35940/ijrte.B1178.0982S919 